(c) 2009 Cambridge University Press

http://journals.cambridge.org/

\title{
Available potential energy diagnosis in a direct numerical simulation of rotating stratified turbulence
}

\author{
Guillaume Roullet ${ }^{1,{ }^{*}}$ and Patrice Klein ${ }^{1}$ \\ ${ }^{1}$ Laboratoire de Physique des Océans UMR6523 (CNRS, UBO, IFREMER, IRD), Brest, France \\ *: Corresponding author : G. Roullet, email address : Guillaume.Roullet@univ-brest.fr
}

\begin{abstract}
:
Review of three studies devoted to the available potential energy (APE) leads to the proposal of a diagnosis for APE, well-suited for rotating stratified flows within the primitive equations (PE) framework in which anharmonic effects (due to large vertical displacements of isopycnals) are permitted. The chosen diagnosis is based on the APE definition of Holliday \& McIntyre (J. Fluid Mech., vol. 107, 1981, pp. 221-225) and uses the background stratification of Winters et al. (J. Fluid Mech., vol. 289, 1995, pp. 115-128). Subsequent evaluation of the APE in a PE direct simulation $\left(1 / 100^{\circ}, 200\right.$ levels) of oceanic mesoscale turbulence indicates that anharmonic effects are significant. These effects are due to large vertical displacements of the isopycnals and the curvature of the background density profile.
\end{abstract}

\section{Introduction}

For quasi-geostrophic (QG) stratified rotating turbulent flows, characterized by small vertical displacements of isopycnals, Charney (1971) predicted that one third of the total energy is in potential form, in accordance with the energy equipartition principle. Such equipartition has been confirmed in numerical simulations of 3-D QG turbulence (Hua \& Haidvogel 1986; McWilliams 1989). In these flows the APE diagnosis has a quadratic form based on the smallness of the isopycnal vertical displacements. There is no equivalent theory for the PE framework which allows flow regimes with large vertical displacements of isopycnals. Actually the potential energy is rarely used and in any case is never diagnosed in PE numerical simulations. This is because of the lack of an adequate APE diagnosis that would take into account the non-quadratic, or anharmonic, effects of APE, which requires to consider higher order terms. In this paper we review in section 2 some studies devoted to the definition of APE. Their synthesis allows us to propose a more accurate APE definition for the PE framework. Such diagnosis is used in section 3 to analyse the APE properties in a PE direct numerical simulation (DNS) and in particular the anharmonic effects. Conclusions are offered in the last section.

\section{A review on APE density}

\subsection{The three definitions of the APE density}

The potential energy of a fluid parcel with density $(x, y, z, t)$ is $\operatorname{ep}(z, \quad)=g z$ (with $g$ the gravity constant). This energy is never diagnosed because it is neither quadratic nor linear in the perturbation. 


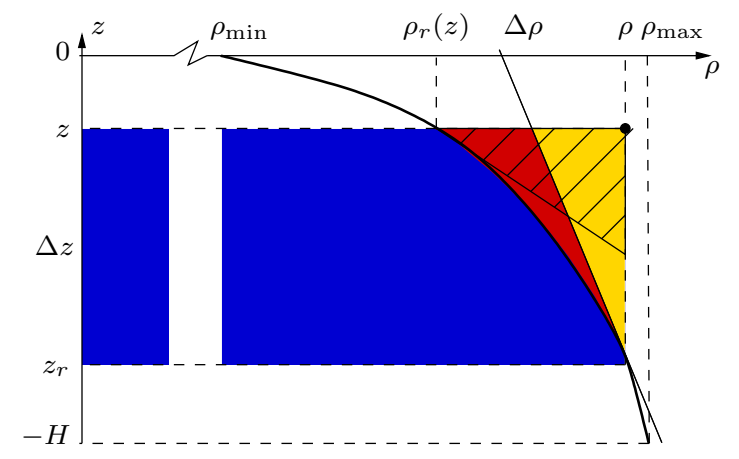

FiguRE 1. Graphical interpretation of the three APE densities in terms of areas, all based on a reference stratification $\rho_{r}(z)$ (thick line): Holliday \& McIntyre's APE (2.1) of a parcel $\rho$ at depth $z$ is the sum of yellow and red areas, the basic APE (2.2) for the same parcel is the sum of the blue, red and yellow areas and QG APE (2.5) is the yellow area whereas QG APE (2.6) is the hatched area. (2.5) is based on the slope of the reference profile $N_{r}^{2}\left(z_{r}\right)$ (thin straight line) and (2.6) on the slope of the profile at $z$. Anharmonic effects (taken into account in (2.1) and not in (2.5)) concern the red area.

A better quantity to use is the APE density proposed by Holliday \& McIntyre (1980) that measures the potential energy with respect to a reference state

$$
e_{a}(z, \rho)=\int_{z_{r}(\rho)}^{z} g\left(\rho-\rho_{r}\left(z^{\prime}\right)\right) d z^{\prime}
$$

where $\rho_{r}(z)$ is the density profile of this reference state and $z_{r}(\rho)$ is its inverse mapping. Physically, $z_{r}(\rho)$ is the equilibrium depth of a parcel of density $\rho$. (2.1) includes two terms. The first one, $g \rho \Delta z$ (with $\Delta z=z-z_{r}(\rho)$, the vertical displacement of the isopycnals), is the work of the gravitational force (see Holliday \& McIntyre 1980) and the second, $\int_{z_{r}(\rho)}^{z} \rho_{r}\left(z^{\prime}\right) g d z^{\prime}$, is the work of the pressure force due to the background stratification. A graphical interpretation of this APE is given in Fig. 1 as the sum of the red and yellow areas. It is actually the area delimited by the curve $z_{r}(\rho)$ and the horizontal and vertical lines emanating from the point $(z, \rho)$. This APE definition requires no assumption on $\Delta z$. One important constraint for this definition is that $z_{r}(\rho)$ must exist for any $\rho$-value present in the fluid and therefore the reference state should span the interval $[\min (\rho), \max (\rho)]$. As noted by Holliday \& McIntyre (1980), this APE density is not quadratic in the perturbation because it includes higher order corrections, namely anharmonic terms, but it has the essential property of being definite positive as long as the reference profile is stable.

A more basic expression for the APE density is

$$
e_{W}(z, \rho)=g \rho \Delta z
$$

that retains only the gravitational force term of the APE expression (2.1). It was used by Winters et al. (1995) to obtain volume integrated APE. This basic APE is the sum of the blue, red and yellow areas on Fig. 1. If $\rho_{b}(z)$ is a volume-preserving rearrangement of $\rho(x, y, z)$ (as in Winters et al.) then the two APEs yield the same domain integrated value

$$
E_{a}[\rho]=\int_{V} e_{a}(z, \rho(x, y, z, t)) d V=\int_{V} e_{W}(z, \rho(x, y, z, t)) d V
$$

However, if one is interested in the local APE density, then only (2.1) is valid because the additional work of the pressure force (blue area on Fig. 1), at leading order in $\Delta z$, 
cancels out the gravitational force (the sum of red, yellow and blue areas on Fig. 1) and transforms the linear form (2.2) into the definite positive form (2.1) while preserving (2.3). The Hamiltonian approach (§ 2.2) provides the unifying framework.

A further simpler and classical expression for the APE is the one that uses, for a fixed density, a Taylor series expansion of (2.1) in $\Delta z=z-z_{r}(\rho)$ (assuming the smallness of $\Delta z)$, that is

$$
e_{a}(z, \rho)=-g\left(\frac{1}{2} \partial_{z} \rho_{r} \Delta z^{2}+\frac{1}{6} \partial_{z z}^{2} \rho_{r} \Delta z^{3}\right)+O\left(\Delta z^{4}\right)
$$

The second order term involves the density gradient and the third order one the curvature of the reference density profile. If only the second order term is retained (2.4) yields the QG APE density (Pedlosky 1987) that is quadratic and reads

$$
e_{Q G}(z, \rho)=\frac{1}{2} \rho_{0}\left[N_{r}\left(z_{r}\right) \Delta z\right]^{2}
$$

where $\rho_{0}$ is the constant density associated with the Boussinesq assumption, and $N_{r}^{2}\left(z_{r}\right)=$ $-\rho_{0}^{-1} g \partial_{z} \rho_{r}\left(z_{r}\right)$ is the square of the Brunt-Väisälä frequency of the reference stratification. The right triangle approximating the QG APE is the yellow area on Fig. 1 where the hypothenuse is given by the local slope $N_{r}^{2}\left(z_{r}\right)$ of the profile. (2.1) turns out to be the finite amplitude form of (2.5) and therefore a more accurate definition of APE when $\Delta z$ is large. Conversely, expanding (2.1) in $\Delta z$, at fixed $z$, yields the QG APE density written for the density perturbation $\Delta \rho=\rho-\rho_{r}(z)$

$$
e_{Q G}^{*}(z, \rho)=\frac{1}{2} \rho_{0}\left[\frac{g \Delta \rho}{\rho_{0} N_{r}(z)}\right]^{2}
$$

(hatched right triangle on Fig. 1). For a non uniform reference profile (2.5) and (2.6) differ. In particular, the slopes of the triangles are different. (2.5) is associated with the Lagrangian view (fixed $\rho$ ) whereas (2.6), the most customary, is associated with the Eulerian view (fixed $z$ ).

We define anharmonic effect by

$$
e_{a n h}(z, \rho)=e_{a}(z, \rho)-e_{Q G}(z, \rho)
$$

(red area on Fig 1) and at leading order in $\Delta z$ it is proportional to $\Delta z^{3}$ and to the curvature of the reference profile, $\partial_{z z}^{2} \rho_{r}$, (see (2.4) and (2.5)). Using $e_{Q G}^{*}$ in (2.7) instead of $e_{Q G}$ would give an anharmonic effect with opposite sign and a slightly different magnitude.

The choice of (2.1) was validated by Shepherd (1993) and Bannon (2003) who furthermore explored the role of the pressure forces with the help of the Hamiltonian formalism as described in section 2.2. Then the only question to address is the choice of the reference stratification. This point is discussed in sections 2.3 and 2.4. The next issue will be to determine how (2.5) differs from (2.1) in a highly ageostrophic regime that exhibits large isopycnal deviations and therefore to quantify the importance of the anharmonic effects. These effects, shown by the red area on Fig. 1, are quantified in section 3.

\subsection{Hamiltonian formalism}

We follow in this section some ideas developed in Shepherd (1993) to better understand the role of the pressure force. Prior to the APE definition, two density functionals are introduced: the total potential energy

$$
E_{p}[\rho]=\int_{V} \rho(x, y, z, t) g z d V
$$


and a Casimir which, for a rest state, reduces to a functional of the density

$$
C[\rho]=\int_{V} f_{r}(\rho) d V,
$$

where $f_{r}(\rho)$ is a function defined on the reference state (see Morrison 1998, for a good introduction on Hamiltonian fluid dynamics). This Casimir is chosen so as to cancel the linear contributions in the perturbation. The total APE then reads

$$
E_{a}[\rho]=E_{p}[\rho]-E_{p}\left[\rho_{r}\right]+C[\rho]-C\left[\rho_{r}\right],
$$

which is also named the potential part of the pseudo-energy. In order for the Casimir to have no impact on the global form of energy, i.e. $C[\rho]=C\left[\rho_{r}\right], \rho_{r}(z)$ must be a volumepreserving rearrangement of $\rho(x, y, z)$. This sets a strong constraint on the reference profile. In this case, the introduction of the Casimir only modify the APE local form. Shepherd (1993) gives an extensive review of the various APE associated with the various set of fluid dynamics equations. Applied to the case of stratified rotating incompressible flows, hydrostatic or not, the Casimir reads

$$
f_{r}(\rho)=-p_{r}\left(z_{r}(\rho)\right)-\rho g z_{r}(\rho),
$$

where $p_{r}(z)$ is the hydrostatic pressure associated with the reference stratification. Hence, the APE density reads

$$
e_{a}(z, \rho)=\rho g z-\rho g z_{r}(\rho)-p_{r}\left(z_{r}(\rho)\right)+p_{r}(z)
$$

recovering readily (2.1). The two first terms of the right-hand side of (2.12) give (2.2), the two next terms correspond physically to the work of the pressure force. The cancellation of the linear component by the Casimir is illuminating in (2.12): indeed, at leading order $p_{r}(z)-p_{r}\left(z_{r}\right) \sim-\rho g\left(z-z_{r}(\rho)\right)$. To summarize: $e_{W}$ is the work of the gravity force, $e_{Q G}$ and $e_{Q G}^{*}$ include the leading order term of the pressure forces work and $e_{a}$ includes the exact work of pressure forces (Fig 1).

\subsection{Choice of the reference stratification}

The choice of a reference stratification $\rho_{r}(z)$ in $(2.1)$ is a priori arbitrary provided that $z_{r}(\rho)$ exists for any $\rho$-value present in the fluid. Incidentally, the use of the customary horizontally averaged profile $\bar{\rho}(z)$ is not in general possible because parcels at surface may have density less than the surface mean density $\bar{\rho}(z=0)$ (which is the minimum value for the reference stratification). However, if we want APE to be the maximal potential energy that can be extracted from a given mass field in an adiabatic way, then, the reference stratification must be the so-called background stratification $\rho_{b}(z)$ (Lorenz 1955). It is basically the flat stratification obtained by an adiabatic rearrangement of the parcels. Therefore, a reference profile is univocally associated with any given state, though at the expense of a highly implicit fonction. For the case of an incompressible equation of state, this function is simply a sorting of parcels on their density (Winters et al. 1995). The background stratification ensures that the Casimirs contributions globally vanish and that (2.3) holds. It is worth noting that other reference stratifications could be used, but then APE would loose its simple physical interpretation. For a forced-dissipative flow, the concept of background stratification still makes sense because it can be defined in terms of statistical properties of the density (cf. $\S 2.4$ ) and not in terms of an adiabatic transformation.

To our knowledge, the APE definition of Holliday \& McIntyre (1980) - i.e. (2.1) - has never been diagnosed in models of rotating stratified turbulence. The central goal of this paper is therefore to study the properties of (2.1) in a DNS of a turbulent flow with 
the choice $\rho_{r}(z)=\rho_{b}(z)$. Before, let us further explore the properties of this background stratification.

\subsection{The background stratification}

By definition, the background stratification $\rho_{b}(z)$ is the density field associated with a given state $\rho(x, y, z, t)$ that minimizes the potential energy under adiabatic displacements of parcels. Under the incompressible assumption, the background stratification is also the cumulative probability density function (pdf) of the density as shown below. Let us introduce the following two cumulative pdfs: the volume $V(\rho)$ occupied by parcels lighter than $\rho$

$$
V(\rho)=\int_{\rho^{\prime}<\rho} d V^{\prime},
$$

and $V(z)$ the volume of water above depth $z$

$$
V(z)=\int_{z^{\prime}>z} d V^{\prime} .
$$

The derivatives of each of these function $\partial_{\rho} V$ and $\Sigma(z)=-\partial_{z} V$ are respectively the pdf of the density weighted by the volume and the surface of the ocean at depth $z$. Since the function $V(z)$ is monotonic, its inverse mapping $z(V)$ exists. Composing $z(V)$ and $V(\rho)$ yields to the inverse mapping of the background stratification

$$
z_{b}(\rho)=z(V(\rho))
$$

which is the cumulative pdf of the density. Using the chain rule yields

$$
\frac{\partial z_{b}}{\partial \rho}(\rho)=-\Sigma(z)^{-1} \frac{\partial V}{\partial \rho}(\rho),
$$

which is the density pdf weighted by the thickness, that simplifies, in the case of a domain with a flat bottom, into

$$
N_{b}^{2}\left(z_{b}(\rho)\right)=-\frac{g \Sigma_{0}}{\rho_{0}}\left[\partial_{\rho} V(\rho)\right]^{-1}
$$

where the surface $\Sigma_{0}=\Sigma(z)$ is independent of $z$. Therefore, in practice, computing $N_{b}^{2}\left(z_{b}(\rho)\right)$ amounts to compute the density pdf $\partial_{\rho} V(\rho)$.

\section{Model results}

\subsection{Description}

Numerical simulations of a nonlinear baroclinic unstable flow in a zonal $\beta$-plane channel (1000 km long, $3000 \mathrm{~km}$ wide and with a depth of $4000 \mathrm{~m})$ centered at midlatitudes $(f=$ $10^{-4} s^{-1}$ ), have been performed with the Primitive Equations code ROMS (see details in Klein et al. (2008)). The simulation used in this paper has a $1 \mathrm{~km}$ horizontal resolution, corresponding roughly to $1 / 100^{\circ}$ resolution, and 200 vertical levels concentrated at sea surface whose thickness is exponentially increasing with depth. The simulation, forced by a linear restoring (50 days) of its mean zonal state to a prescribed climatological state (Fig. 2), is integrated until the statistical equilibrium is reached (600 days from the original zonal state triggered by a small random noise). Upper layer dynamics is further explored by Klein et al. (2008).

\subsection{Background stratification}

Fig. 2 highlights that the horizontally averaged density profile, $\bar{\rho}$, is very different from the background density profile, $\rho_{b}$, in the upper layers but the two coincide in the abyss. 

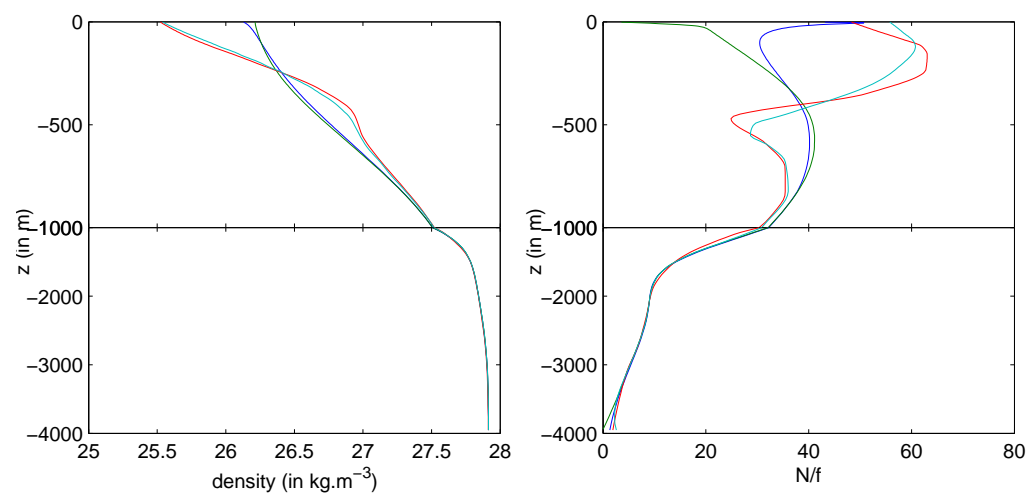

Figure 2. (Left) Vertical profiles (with a zoom in the upper layers) of: the horizontally averaged density $\bar{\rho}$ at the equilibrium (blue), and of the horizontally averaged climatology density $\rho_{\text {clim }}$ (green) and their associated background profiles $\rho_{b}$, (red) and $\rho_{b \text { clim }}$ (cyan) (Right) $N / f$ (dimensionless) profiles associated with the different density profiles (identified by their color). The increase of $N / f$ at surface associated to $\bar{\rho}$ is an evidence of the restratification process at play in this run.
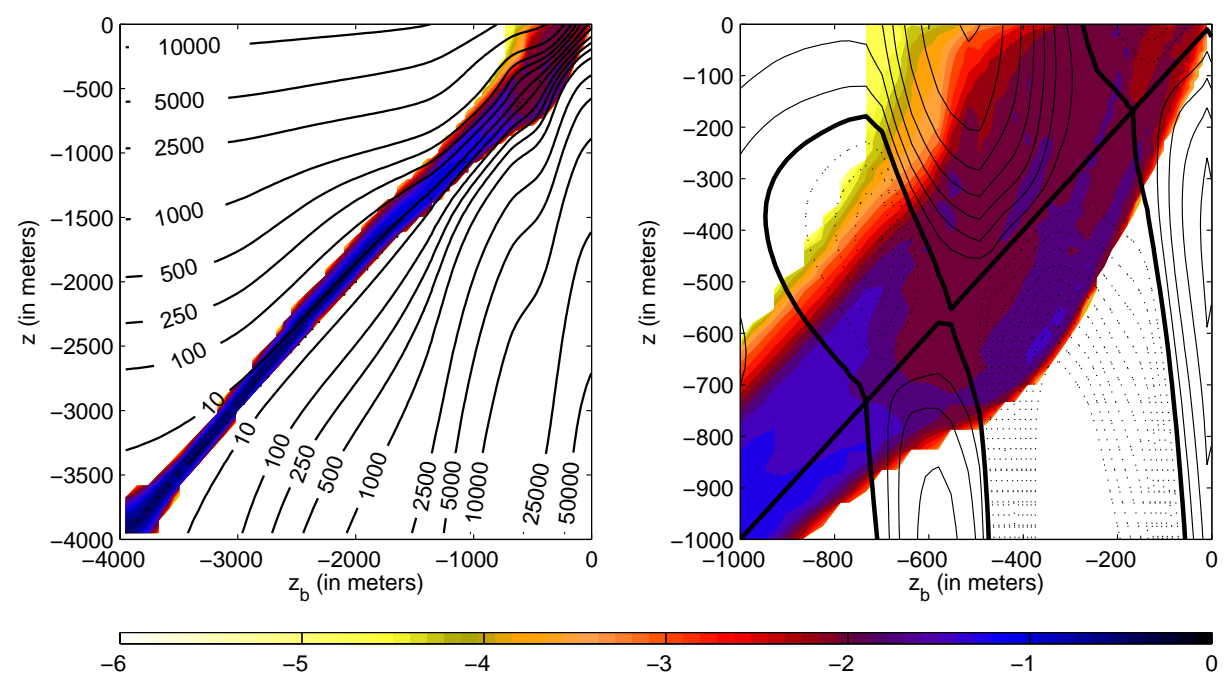

FIGURE 3. Left: thin iso-contours of $\operatorname{APE} \tilde{e}_{a}\left(z, z_{b}\right)$ superimposed on the joined-pdf of the APE (log scale in color) expressed as a function of $z$ and $z_{b}$. Right: zoom on the upper-right corner with relative importance of the anharmonic effects $e_{a n h} / e_{a}$ (iso-contours every 0.1 , dashed for negative values, thick for 0 value) superimposed on the same colored pdf. Isocontours are solely determined by $\rho_{b}(z)$, whereas colors result from the $3 \mathrm{D}$ oceanic turbulent simulation. When looking at constant $z_{b}$ (i.e. on a given isopycnal), the joined-pdf provides the pdf of the depth $z$ of this isopycnal: for instance the $z_{b}=400 \mathrm{~m}$ isopycnal spreads from roughly $800 \mathrm{~m}$ depth to the surface.

However, when vertically integrated, these two profiles yield the same mass. Furthermore the background stratification $\left(\rho_{b}\right)$ is close to the one $\left(\rho_{b}^{*}\right)$ calculated from the climatological state (Fig. 2). There is no physical necessity because the forcing is basically diabatic and so may modify the background stratification. This property is due to the particular choice of forcing that drives continuously the density toward the climatology. 

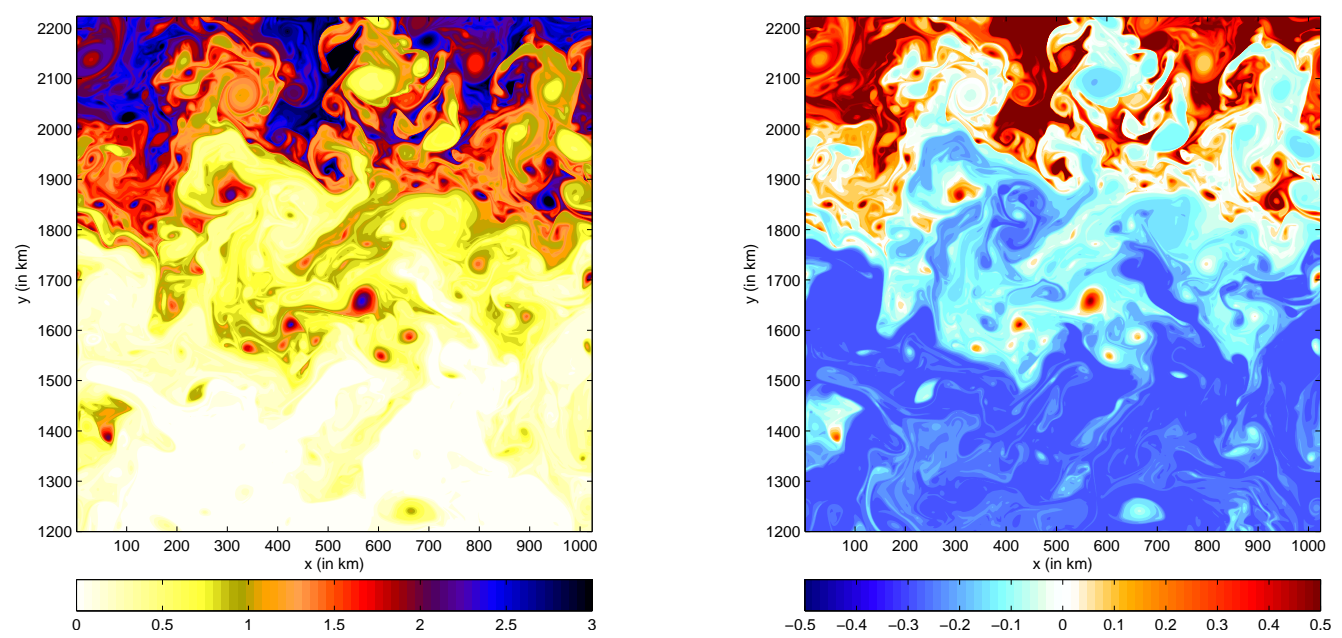

Figure 4. Left: snapshot at surface in the central region of the jet of APE density $e_{a} / \rho_{0}$ (in $m^{2} . s^{-2}$ ). Right: relative importance of the anhharmonic effects, $e_{a n h} / e_{a}$ (dimensionless), estimated from (2.1) and (2.7).

\subsection{Statistical properties of APE}

Because $z_{r}(\rho)$ is monotonic, the APE density can be expressed in terms of either $(z, \rho)$ or $\left(z, z_{b}\right)$ using a composition, namely $\tilde{e}_{a}\left(z, z_{r}\right)=e_{a}\left(z, \rho_{r}\left(z_{r}\right)\right)$ with

$$
\tilde{e}_{a}\left(z, z_{b}\right)=\int_{\rho_{b}(z)}^{\rho_{b}\left(z_{b}\right)}\left(z-z_{b}\left(\rho^{\prime}\right)\right) g d \rho^{\prime} .
$$

The structure of the APE for a fluid parcel in the parameter space $\left(z, z_{b}\right)$ is sketched by the isocontours on Figure 3a. The zero contour is along the diagonal. In the vicinity of the diagonal $\tilde{e}_{a}\left(z, z_{b}\right)$ is locally quadratic in the transverse direction, which means that anharmonic effects vanishes along the diagonal (Fig. 3b) and that the APE matches the QG APE. For large vertical displacements $\left(|\Delta z|=\left|z-z_{b}\right|\right)$ APE deviates from a harmonic potential, which is the signature of the anharmonic effects.

The joint pdf of the APE from the ocean turbulent eddy field (superimposed in colorscale on Figure 3) has been computed by scanning every model grid-cell, associating with each the pair $\left(z, z_{b}(\rho)\right)$, then counting the number of grid-cells with a given $\left(z, z_{b}(\rho)\right)$. We have a total of $6 \times 10^{8}$ grid cells in the simulation domain. Below $500 \mathrm{~m}$ the pdf peaks around the main diagonal with a small transverse width. For upper layers, above $500 \mathrm{~m}$, the pdf peak well deviates from the diagonal with deviation increasing as $z$ tends to 0 . This deviation illustrates the anharmonic effects in the numerical simulation due to the large $\Delta z$. These effects are important (locally $70 \%$ of the total APE) at the surface where relatively dense water outcrop, corresponding to vertical displacements of up to $800 \mathrm{~m}$. Interestingly, they are also important below the thermocline, at $600 \mathrm{~m}$ depth, where they exceed $70 \%$ of the total APE. The sign of anharmonic effect at $600 \mathrm{~m}$ depth (Fig. 3b) is directly related to the convexity of the background profile (Fig. 2).

\subsection{APE in the physical space}

At fixed $z, \mathrm{APE}$ is a functional of density only and at leading order is captured by the QG-like expression (see (2.5) or (2.6)). However, deviations from a purely QG APE are not so small (Fig. 4b) and reach $50 \%$ where density anomalies are large. Anharmonic effects are negative (positive) in the south (north) part of the domain where the vertical 
$\rho$
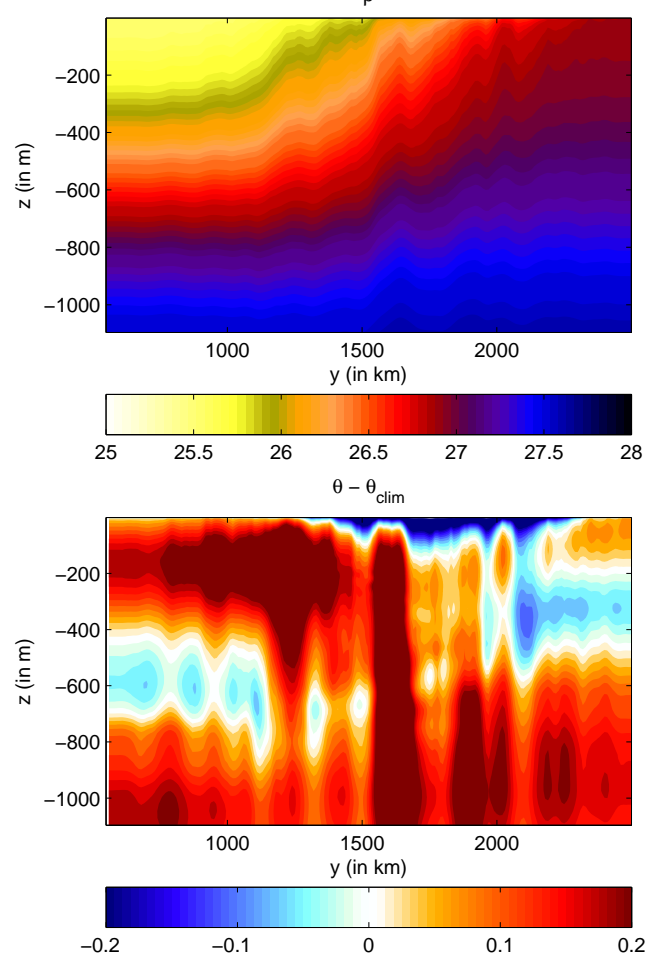

$\theta$
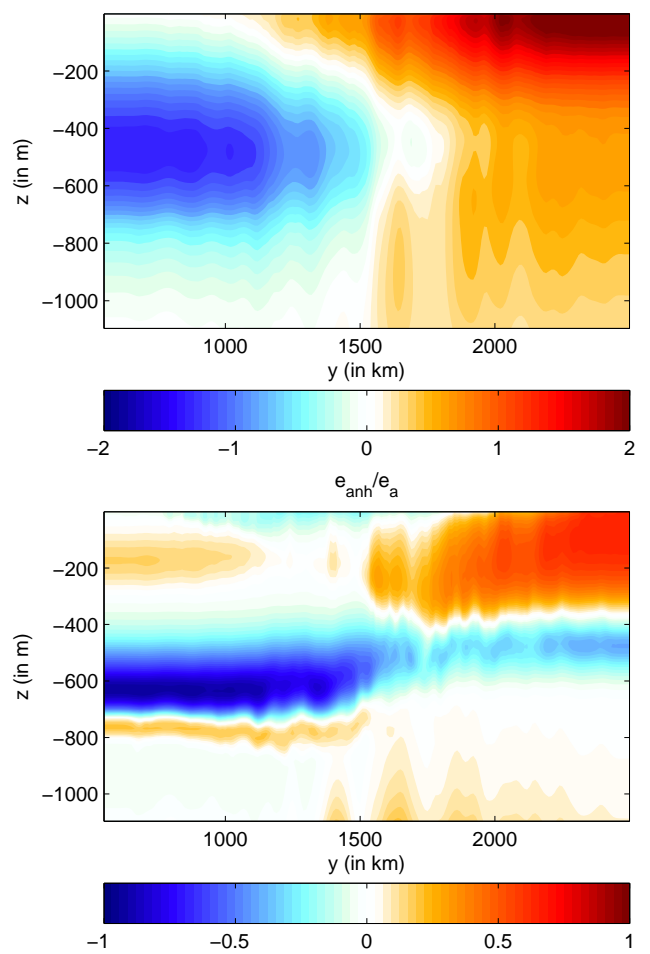

Figure 5. Zonal mean snapshots in the central part of the channel of $\rho$ (a in $\left.\mathrm{kg}^{-3} \mathrm{~m}^{-3}\right), \theta$ (b in $\left.m . s^{-1}\right), \theta-\theta_{\text {clim }}\left(\mathrm{c}\right.$ in $\left.m . s^{-1}\right)$ and $e_{a n h} / e_{a}$ (d, dimensionless).

displacements of the isopycnals are small (large). This is consistent with the isocontours of (Fig. 3b) that displays a small negative region in the upper right and a positive region in the upper middle. At deeper level, anharmonic effect continues to be of the same order as at the surface. It is only below $800 \mathrm{~m}$ depth that anharmonic effect drops to few percent and so that APE can be considered as the square of the density anomaly.

To further investigate how APE varies on the vertical we define an equivalent perturbation for the APE density

$$
\theta \widehat{=} \frac{\Delta z}{|\Delta z|} \sqrt{\frac{2 e_{a}}{\rho_{0}}}
$$

that can be either positive $(\Delta z>0)$ or negative $(\Delta z<0)$, and that can be compared with the QG perturbation

$$
\theta_{Q G}=N_{b}\left(z_{b}(\rho)\right)\left[z-z_{b}(\rho)\right] .
$$

With these definitions, APE densities read $e_{a}=\rho_{0} \theta^{2} / 2$ and $e_{Q G}=\rho_{0} \theta_{Q G}^{2} / 2$.

On a zonal mean section, APE has nothing common with density, though it is completely related to it. On one hand density (Figs. 5a) reflects the structure of the mass field, with classical features: a thermocline, outcropping of isopycnals, meridional gradient. On the other hand, $\theta$ reflects the primary source of energy of the flow linked to the density anomalies (Fig. 5b). Indeed, both the minimum and the maximum of $\theta$ located at depth on the south and north flank of the jet are associated with Ertel PV extrema (not shown) leading to a strong meridional PV gradient that is responsible for the persistency of the baroclinic instability conditions. These extremas are sustained by 

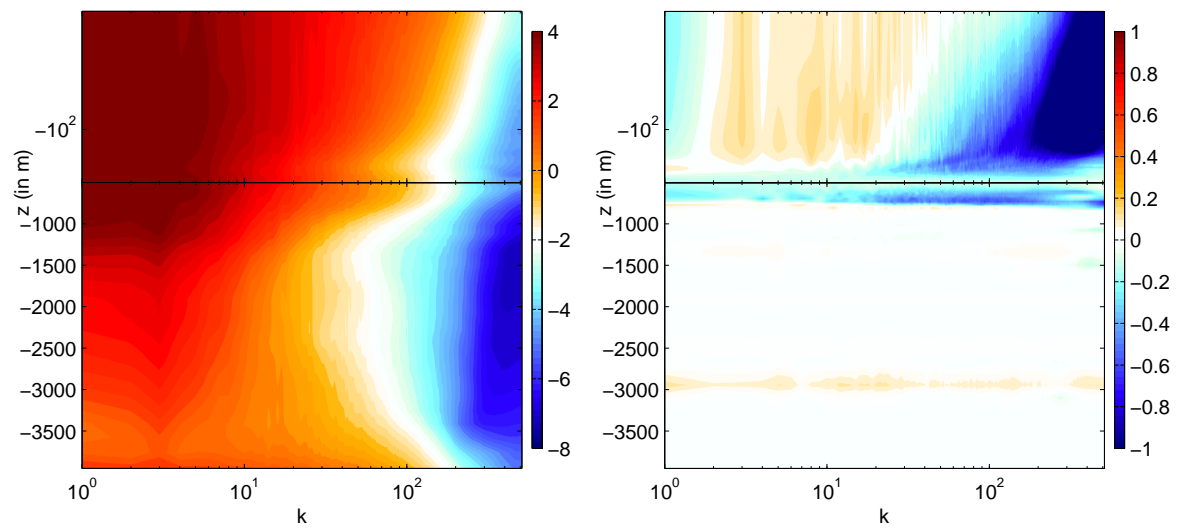

FIGURE 6. Left: APE horizontal spectrum (isocontours of $\log _{10} \widehat{\theta}^{2}$ ) as a function of depth. Upper $500 \mathrm{~m}$ are in log-scale in order to emphasize the vertical exponential decay near the surface Right: Similar to the left panel but for the ratio of QG APE on APE (isocontours of $\log _{10}\left(\widehat{\theta}^{2} / \widehat{\theta}_{Q G}^{2}\right)$.

a balance between the forcing feeding them and the baroclinic instability relaxing them. One interesting feature is that when the climatology contribution is substracted from $\theta$, the resulting field (Fig. 5c) displays chimney-like vertical structures of APE whose depth extension attains $1000 \mathrm{~m}$ and with a width less than $100 \mathrm{~km}$. This indicates the strong impact of the mesoscale turbulence on the APE.

Fig. 5d reveals that APE is larger than its QG counterpart in the first $300 \mathrm{~m}$ below the surface (except for an area very close to the surface in the southern part of the domain). On the other hand it is smaller than its QG counterpart between $300 \mathrm{~m}$ and $700 \mathrm{~m}$. Again these positive and negative deviations of APE from its QG-expression, that attain more than $50 \%$, are consistent with the isocontours of Fig. 3b that display, on the average, a positive value above $300 \mathrm{~m}$ and a negative value between $300 \mathrm{~m}$ and $700 \mathrm{~m}$. This means that the amplitude and sign of these anharmonics effects are entirely determined by the background density profile and mostly by its curvature as discussed in section 2.1.

\subsection{APE in the spectral space}

The definition of $\theta$ proposed in (3.2) also allows the computation of the APE spectra at different depths.

$$
e_{a}(k, z)=\frac{1}{2} \int_{0}^{2 \pi}|\widehat{\theta}|^{2} k d \varphi
$$

where $\hat{\theta}(k, \varphi, z)$ is the horizontal Fourier transform of $\theta(x, y, z)$ in polar coordinates. Fig. 6a shows the distribution of APE in a spectral space. For a given horizontal scale, the maximum of APE is at surface, because of the surface intensified nature of the turbulence. APE at submesoscale $(k>50)$ is also intensified at surface but decreases rapidly with depth. More precisely, in the upper $100 \mathrm{~m}$, APE isocontours are straight and inclined, indicating that the decay of APE is exponential (because the vertical scale is a log-scale). This exponential decay of surface dynamics is well captured by the surface quasi-geostrophic (SQG) theory. A small exponential decay is also present at the bottom due to a bottom trapped dynamics analog to the SQG dynamics. Below the thermocline, APE decreases rapidly with $k$, indicating a QG regime. These results are in accordance with the results of Klein et al. (2008) that reveal a $k^{-2}$ spectrum slope for the density near the surface instead of a $k^{-4.5}$ spectrum slope in the abyss.

The anharmonic effects have a relatively simple structure in the $(k, z)$ space (Fig. $6 \mathrm{~b}$ ). 
They are mostly negative for large $k$ and positive for smaller $k$ in the upper layers. Again this is consistent with the isocontours of Fig. 3b. Indeed, the Burger number being close to one in this simulation (Klein et al. 2008), small horizontal structures have also a small vertical extension, allowing only small vertical displacements of the isopycnals. The opposite is true for the larger scale structures. Fig. 3b, on the other hand, reveals that anharmonic effects in the upper $300 \mathrm{~m}$ are negative (positive) in the upper right (middle) part where small (large) displacements of the isopycnals are allowed. Below the thermocline, the QG APE is too large, i.e. anharmomic effets are negative, at all scales. Below $1000 \mathrm{~m}$, anharmonic effects are negligible.

\section{Conclusion}

We have confirmed that the APE density as defined by Holliday \& McIntyre (1980) takes into account terms that are missing from the basic and the QG Pedlosky (1987) definition of APE. As such it is the more appropriate within the framework of the primitive equations. This remains true even with the hydrostatic assumption relaxed. Like every APE, it is based on reference density profile. We have shown that not every profile suits. Following Lorenz's (1955) physical interpretation of APE, we used the background density profile obtained by an adiabatic rearrangement of the parcels. We have shown that for an incompressible equation of state, this profile is the cumulative pdf of density. This property allows for the computation of the background stratification in a more complex geometry. Using these results we have estimated the APE density in a DNS of a rotating stratified turbulent flow that uses a PE model. To our knowledge, such estimation in a PE simulation has never been done before. The estimated APE density significantly departs from its QG counterpart because of the strong isopycnal displacements. But this is essentially the curvature of the background density profile that determines the amplitude and the sign of the departures of APE from its QG counterpart, which are called the anharmonic effects. These anharmonic effects are significant principally within the upper oceanic layers.

\section{REFERENCES}

Bannon, P. R. 2003 Hamiltonian description of idealized binary geophysical fluids. J. Atmos. Sci. 60, 2809-2819.

Charney, J. G. 1971 Geostrophic turbulence. J. Atmos. Sci. 28, 1087-1095.

Holliday, D. \& McIntyre, M. E. 1980 On potential energy density in an incompressible stratified fluid. J. Fluid Mech. 107, 221-225.

Hua, B. L. \& Haidvogel, D. B. 1986 Numerical simulations of the vertical structure of quasigeostrophic turbulence. J. Atmos. Sci. 43, 2923-2936.

Klein, P., Hua, B. L., Lapeyre, G., Capet, X., Le Gentil, S. \& Sasaki, H. 2008 Upper ocean turbulence from high 3 -d resolution simulations. J. Phys. Oceanogr. in press.

Lorenz, E. N. 1955 Available potential energy and the maintenance of the general circulation. Tellus 7, 157-167.

MCWilliams, J. C. 1989 Statistical properties of decaying geostrophic turbulence. J. Fluid Mech. 198, 199-230.

Morrison, P. J. 1998 Hamiltonian description of the ideal fluid. Rev. Mod. Phys. 70, 467-521.

Pedlosky, J. 1987 Geophysical Fluid Dynamics. Springer Verlag.

Shepherd, T. G. 1993 A unified theory of available potential energy. Atmos.-Ocean 31, 1-26.

Winters, K. B., Lombard, P. N., Riley, J. J. \& D'Asaro, E. A. 1995 Available potential energy and mixing in density-stratified fluids. J. Fluid Mech. 289, 115-128. 